\title{
Adoption of Sorafenib for the Treatment of Advanced-Stage Hepatocellular Carcinoma in Oncology Practices in the United States
}

\author{
Helen M. Parsons ${ }^{a} \quad$ Quyen Chu $^{b} \quad$ Jordan J. Karlitz ${ }^{c} \quad$ Jennifer L. Stevens ${ }^{d}$ \\ Linda C. Harlan ${ }^{\mathrm{e}}$ \\ a Division of Health Policy and Management, University of Minnesota, Minneapolis, MN, \\ b LSU-Health Sciences Center-Shreveport, Shreveport, LA, ' Division of Gastroenterology, \\ Tulane University School of Medicine, New Orleans, LA, dinformation Management \\ Services, Calverton, MD, and eDivision of Cancer Control and Population Sciences, \\ National Cancer Institute, Bethesda, MD, USA
}

\section{Keywords}

Hepatocellular carcinoma $\cdot$ SEER Program $\cdot$ Policy $\cdot$ Treatment $\cdot$ Sorafenib

\begin{abstract}
Background: The adoption of sorafenib into oncology practice as a first-line systemic treatment for advanced hepatocellular carcinoma (HCC) is not well understood. We examined sorafenib use since Food and Drug Administration (FDA) approval in 2007 and associated survival for individuals diagnosed with advanced HCC, conducting a population-based evaluation of treatment patterns and outcomes for this newly approved drug in the US over time. Methods: We identified individuals diagnosed with Barcelona Clinic Liver Cancer Stage C from the 2007 and 2012 National Cancer Institute Patterns of Care study. We examined trends in use as well as patient and clinical factors associated with receiving sorafenib using multivariate logistic regression analysis. We then evaluated the association between sorafenib use and overall hazard of death using multivariate Cox proportional hazards regression. Results: Among 550 individuals diagnosed with advanced HCC, we found no significant increase in the proportion of patients treated with sorafenib from 2007 to 2012 (26.3 vs. 30.4\%). After adjusting for patient and clinical characteristics, non-Hispanic Blacks (compared to non-Hispanic Whites) and those with a lower Child-Pugh score remained more likely to receive sorafenib. Individuals receiving systemic chemotherapy only, radiation therapy only, or no treatment at all experienced a higher risk of death than those treated with sorafenib, while those receiving a transplant experienced a lower risk of death. Conclusions: Sorafenib has not been widely adopted into oncology practice since FDA approval for advanced HCC. Few factors apart from Child-Pugh score and race/ethnicity predict sorafenib use in clinical practice, although sorafenib treatment is associated with a lower risk of death.

(c) 2017 S. Karger AG, Basel
\end{abstract}




\section{Introduction}

Rates of hepatocellular carcinoma (HCC) have tripled over the past 30 years [1], with more than 35,000 new cases diagnosed annually in the US $[2,3]$. While the incidence of HCC continues to rise, the overall 5 -year survival rate remains extremely low at $<12 \%$ [2]. Though diagnosis with early-stage HCC provides opportunities for several potentially curative treatment options [4], presentation with advanced HCC (Barcelona Clinic Liver Cancer [BCLC] Stage C) was historically met with limited treatment options and poor prognosis [5]. Until recently, there was no systemic therapy demonstrated to effectively treat individuals with advanced HCC [6]. In November 2007, sorafenib, a novel oral multikinase inhibitor, was approved as a first-line systemic treatment for advanced, inoperable HCC by the Food and Drug Administration (FDA) [7]. The approval was based on 2 independent phase III randomized trials demonstrating prolonged median overall survival in individuals with advanced HCC treated with sorafenib compared to placebo [8,9]. As a new molecular-targeted therapy, sorafenib has the potential to provide significantly new treatment choices to those with previously limited options; however, clinical and payer groups remain conflicted about treatment recommendations [10-12].

Since the approval of sorafenib, studies have focused primarily on the safety and efficacy of this new molecular-targeted therapy in single-institution, clinical settings $[5,13,14]$. While these studies have each demonstrated clinical benefit in treating advanced HCC $[5,13,14]$, others note the need to balance this opportunity with the potential for side effects and high treatment costs in the community setting $[15,16]$. Specifically, treatment with sorafenib may cause a higher incidence of adverse drug reactions, such as hand-foot skin reactions, hypertension, and diarrhea, as well as an estimated USD 10,000-15,000/month in drug-related treatment costs $[8,17-20]$. Further, although trials to demonstrate the initial efficacy of sorafenib were limited to well-selected clinical populations [8, 9], the adoption of sorafenib into oncology practice since FDA approval in 2007 is not well understood.

To further understand the adoption of sorafenib and associated survival for individuals diagnosed and treated with advanced HCC in oncology practices, we conducted a populationbased evaluation of treatment patterns and outcomes for this newly approved drug in the US over time. We hypothesized that sorafenib treatment would vary across patient demographic and clinical characteristics and that, consistent with earlier clinical trials, we would find a survival benefit in those treated with sorafenib for advanced HCC in oncology practices.

\section{Materials and Methods}

Data and Sampling

We used the 2007 and 2012 National Cancer Institute (NCI) Patterns of Care data for our study [21], which included a random sample of individuals diagnosed with HCC in the Surveillance, Epidemiology and End Results (SEER) Program registries. The SEER Program is a large set of population-based cancer registries in the US, covering more than $28 \%$ of the US population [22]. SEER currently collects information on individuals' demographics, cancer diagnosis, tumor characteristics, initial treatment, and follow-up for vital status [22]. As the SEER Program is primarily hospital based, adjuvant therapy for cancer treatment provided in an outpatient setting may be underreported. As a result, the NCI annually conducts more comprehensive data collection on cancer treatment for a sample of patients diagnosed in a SEER area with select cancers [21].

After obtaining institutional review board approval, individuals diagnosed with HCC were stratified by sex, age (2007 only), race/ethnicity, and registry, with a random sample taken from each stratum. Individuals diagnosed at $<20$ years of age (due to the small number of individuals diagnosed prior to this age); those diagnosed previously with cancer (except for non-melanoma skin cancer); those diagnosed simultaneously with another cancer (as this may have influenced primary HCC treatment); and those diagnosed at autopsy 
or on the death certificate only (as they would be ineligible for treatment) were ineligible. In order to obtain more stable estimates of racial/ethnic minorities, non-Hispanic Blacks, Hispanics, Asians/Pacific Islanders, and American Indians/Alaskan Natives were oversampled; however, the final sample is representative of the SEER data. Individuals were included in the sample regardless of whether they were entered on a clinical trial protocol for their treatment. After abstractor training was held to ensure consistency of data collection, hospital data on patient demographics, cancer diagnoses, comorbidities, Child-Pugh score, and initial treatment were abstracted, and each person's treating physicians were contacted to verify treatment [23]. Each physician was asked if other physicians provided treatment to the individual, and these physicians were also contacted.

\section{Patients}

We included individuals diagnosed with BCLC Stage C HCC (International Classification of Diseases for Oncology, 3rd edition, topography C22.0 and morphology M8170-75; 8180). Individuals were classified as having BCLC Stage C if the physician-verified disease was categorized as Child-Pugh score A-B with portal invasion, nodal involvement, or metastatic disease [24]. Performance status was not available for use in the classification. After taking a random sample of those diagnosed in the population-based SEER Program registries in 2007 and 2012 using the methodology stated above, our final sample included 550 individuals with newly diagnosed advanced HCC - allowing us to make inferences about treatment patterns and outcomes for this population as a whole.

\section{Key Measures}

Treatment. Treatment was defined as therapies planned or administered for HCC from the medical record or physician report. For our analysis, we categorized individuals as receiving sorafenib (within 12 months of diagnosis), surgery, liver transplant, systemic chemotherapy, radiofrequency ablation, multiple therapies, no treatment, and unknown treatment.

Survival Time. Time to death for 12-month mortality calculations was defined as the number of months from a patient's diagnosis until death or 12 months, whichever came first. Survival is recorded in whole months in the SEER Program registries for confidentiality reasons.

Patient Demographic and Clinical Characteristics. We categorized information on year of diagnosis (2007 or 2012), age at cancer diagnosis (categorized as $<50,50-59,60-69$, and $\geq 70$ years), sex (male or female), race and ethnicity (categorized as non-Hispanic White, non-Hispanic Black, Hispanic, and Asian/ other), and marital status (married/living as married and other). Insurance status was categorized hierarchically from all insurance providers recorded in the medical record into any private insurance (including military), any Medicaid, Medicare only, and no or unknown insurance. Median income for the individual's census tract at diagnosis was categorized as USD $\leq 50,000 /$ year or USD $>50,000 /$ year. Finally, the presence of cirrhosis or hepatitis (A, B, or C) as well as the Child-Pugh score at diagnosis (A or B) were categorized based on documentation of disease by the physician or medical records.

\section{Analysis}

We first conducted bivariate analyses using $\chi^{2}$ tests to examine the unadjusted differences in sorafenib use across patient demographic and clinical characteristics. We then used multivariable logistic regression to examine the association between patient demographic and clinical characteristics and treatment with sorafenib, adjusting for year of diagnosis, age, sex, race/ethnicity, marital status, insurance, median census tract income, hepatitis, cirrhosis, and Child-Pugh score. Finally, we used multivariable Cox proportional hazards regression to examine the association between treatment for advanced HCC and 12-month all-cause mortality, adjusting for all variables included in our logistic regression model as well as treatments received. All estimates from our analyses were weighted using sample weights in order to be representative of the population from which the sample was drawn. The sampling weight is calculated as the inverse of the sampling proportion within each sampling stratum. All analyses were conducted using SAS (SAS Institute, Cary, NC, USA) and SUDAAN (RTI International, Research Triangle Park, NC, USA) statistical software in order to allow for sample weights to adjust standard errors appropriately. All $p$ values were two-sided, with a $p<$ 0.05 considered significant. 


\section{Results}

We identified a sample of 550 individuals diagnosed with advanced HCC in the 2007 and 2012 NCI Patterns of Care Study. The majority of individuals were diagnosed in 2012 (60.4\%), over 60 years of age (54.0\%), male (83.5\%), and non-Hispanic White (53.9\%; Table 1$)$. Further, most individuals were married, were insured under private insurance $(53.1 \%)$, and had a median income of approximately USD 50,000. Clinically, in our sample of advanced HCC patients, the majority had been diagnosed with hepatitis (52.6\%) and cirrhosis (60.0\%) and were classified as Child-Pugh score Class B (63.0\%; Table 1). Of the 309 individuals with Child-Pugh score Class B, 45.8\% had metastatic disease and $57.0 \%$ had portal invasion (data not shown).

\section{Sorafenib Use across Patient and Clinical Characteristics}

Overall, 28.8\% ( $n=186$ ) of individuals were treated with sorafenib in 2007 or 2012 (Table 1). However, we found no significant increase in the proportion of patients treated with sorafenib from 2007 to 2012 (26.3 vs. 30.4\%, respectively; $p=0.37$ ). In unadjusted analyses, we found significant variation in sorafenib use by race/ethnicity (39.5\% of Asians/ other race/ethnicity, $32.8 \%$ of Hispanics, $31.7 \%$ of non-Hispanic Blacks, and $23.6 \%$ of nonHispanic Whites; $p=0.01)$, insurance status $(34.2 \%$ of privately insured vs. $17.6 \%$ of uninsured/unknown insurance status; $p=0.04)$, and Child-Pugh score (39.7\% Class A vs. $22.4 \%$ Class $B ; p=0.0004$ ). No significant differences in sorafenib use were found for age at diagnosis, sex, marital status, median census tract income, hepatitis, or cirrhosis status (Table 1).

After adjusting for patient and clinical characteristics, we continued to find no significant increase in the use of sorafenib over time (Table 2). However, non-Hispanic Blacks (odds ratio $1.89,95 \%$ confidence interval [CI] 1.03-3.46 vs. non-Hispanic Whites) and those with a lower Child-Pugh score (odds ratio $0.46,95 \%$ CI $0.27-0.79$ for Class B vs. A) remained more likely to receive sorafenib (Table 2).

\section{2-Month Mortality}

More than 79.6\% $(n=439)$ of patients with advanced HCC in our study had died by 12 months after diagnosis. The median survival was 7 months in those treated with sorafenib compared to 3 months in those receiving other treatments. After taking patient and hospital factors into account, we found that the type of treatment patients received was highly associated with the 12-month relative hazard of death (Table 3). Specifically, individuals receiving systemic therapy only (median survival 3 months), radiofrequency ablation only (median survival 2.5 months), or no treatment (median survival 2 months) all experienced a higher risk of death than those treated with sorafenib (median survival 7 months), while those receiving a transplant experienced a lower risk of death (median survival not reached at 12 months) ( $p<0.05$ for all). Further, we found that patients who were older at diagnosis or had a Child-Pugh score Class B (hazard ratio 1.43, 95\% CI 1.07-1.90 vs. Class A) all had a higher hazard of 12-month mortality.

\section{Discussion}

In our population-based study of 550 individuals treated for advanced HCC, we found that sorafenib treatment has not been widely adopted into oncology practice. Only $30.4 \%$ of individuals were treated with sorafenib in 2012, which is a nonsignificant increase in use since FDA approval in 2007. Few demographic or clinical factors apart from a lower ChildPugh score and race/ethnicity predicted sorafenib use in clinical practice; however, consistent with phase III clinical trials [8,9], we demonstrate that sorafenib treatment in oncology prac- 


\section{Liver Cancer}

\begin{tabular}{l|l}
\hline \multicolumn{2}{l}{ Liver Cancer 2017;6:216-226 } \\
\hline DOI: 10.1159/000473862 & $\begin{array}{l}\text { C 2017 S. Karger AG, Basel } \\
\text { www.karger.com/lic }\end{array}$ \\
\hline
\end{tabular}

Parsons et al.: Adoption of Sorafenib for the Treatment of Advanced-Stage Hepatocellular Carcinoma in Oncology Practices in the United States

Table 1. Sorafenib use across demographic and clinical characteristics of individuals diagnosed with advance HCC in community settings (2007 and 2012 National Cancer Institute Patterns of Care data)

\begin{tabular}{|c|c|c|c|}
\hline & $\begin{array}{l}\text { All patients, } \\
n(\%)\end{array}$ & $\begin{array}{l}\text { Sorafenib } \\
\text { use row, } \%^{a}\end{array}$ & $\begin{array}{l}p \\
\text { value }\end{array}$ \\
\hline Total & 550 & $186(28.8 \%)$ & \\
\hline Year of diagnosis & & & 0.37 \\
\hline 2007 & $172(39.6)$ & 26.3 & \\
\hline 2012 & $378(60.4)$ & 30.4 & \\
\hline Age at diagnosis & & & 0.86 \\
\hline$<50$ years & $56(10.6)$ & 23.8 & \\
\hline 50-59 years & $146(35.4)$ & 27.9 & \\
\hline 60-69 years & $183(31.1)$ & 31.7 & \\
\hline$\geq 70$ years & $165(22.9)$ & 28.5 & \\
\hline Sex & & & 0.20 \\
\hline Male & $415(83.5)$ & 29.6 & \\
\hline Female & $135(16.5)$ & 24.8 & \\
\hline Race/ethnicity & & & 0.01 \\
\hline Non-Hispanic White & $238(53.9)$ & 23.6 & \\
\hline Non-Hispanic Black & $119(13.7)$ & 31.7 & \\
\hline Hispanic & $91(16.3)$ & 32.8 & \\
\hline Asian/other & $102(16.1)$ & 39.5 & \\
\hline Marital status & & & 0.08 \\
\hline Married/living as married & $291(53.1)$ & 33.5 & \\
\hline Other & $259(46.9)$ & 23.4 & \\
\hline Insurance status & & & 0.05 \\
\hline Private insurance & $304(53.1)$ & 34.2 & \\
\hline Medicare only & $64(10.2)$ & 25.6 & \\
\hline Any Medicaid & $149(30.8)$ & 22.6 & \\
\hline No insurance/unknown & $33(5.9)$ & 17.6 & \\
\hline Median census tract income & & & 0.06 \\
\hline USD $\leq 50,000 /$ year & $264(46.0)$ & 23.4 & \\
\hline USD $>50,000 /$ year & $286(54.0)$ & 33.4 & \\
\hline \multicolumn{4}{|c|}{ Other treatments received (among those without sorafenib use) } \\
\hline Surgery & $13(3.0)$ & n.a. & n.a. \\
\hline Liver transplant & $18(3.1)$ & & \\
\hline Embolization & $27(5.1)$ & & \\
\hline Systemic chemotherapy & $25(5.9)$ & & \\
\hline Radiofrequency ablation & $<11(2.9)$ & & \\
\hline Multiple therapies ${ }^{\mathrm{b}}$ & $58(15.1)$ & & \\
\hline No additional treatment & $203(62.5)$ & & \\
\hline Unknown & $12(2.4)$ & & \\
\hline Hepatitis & & & 0.96 \\
\hline Yes & $305(52.6)$ & 28.9 & \\
\hline No indication & $245(47.4)$ & 28.6 & \\
\hline Cirrhosis & & & 0.06 \\
\hline Yes & $300(60.0)$ & 24.8 & \\
\hline No indication & $250(40.0)$ & 34.7 & \\
\hline \multicolumn{4}{|l|}{ Child-Pugh score } \\
\hline Class A & $241(37.0)$ & 39.7 & \\
\hline Class B & $309(63.0)$ & 22.4 & 0.0004 \\
\hline
\end{tabular}

HCC, hepatocellular carcinoma; n.a., not applicable. ${ }^{a}$ Number of patients suppressed per data requirements, as cell counts were $<11$ in some cases. ${ }^{b}$ Multiple therapies included any combination of treatment with surgery, liver transplant, embolization, systemic chemotherapy, or radiofrequency ablation. 


\section{Liver \\ Cancer}

Table 2. Association between patient demographic and clinical characteristics and sorafenib use, multivariable logistic regression (2007 and 2012 National Cancer Institute Patterns of Care data)*

\begin{tabular}{l|l}
\hline Liver Cancer 2017;6:216-226 \\
\hline DOI: 10.1159/000473862 & $\begin{array}{l}\text { @ 2017 S. Karger AG, Basel } \\
\text { www.karger.com/lic }\end{array}$ \\
\hline
\end{tabular}

Parsons et al:: Adoption of Sorafenib for the Treatment of Advanced-Stage Hepatocellular Carcinoma in Oncology Practices in the United States

\begin{tabular}{|c|c|}
\hline & $\begin{array}{l}\text { Odds ratio ( } 95 \% \\
\text { confidence interval) }\end{array}$ \\
\hline \multicolumn{2}{|l|}{ Year of diagnosis } \\
\hline 2007 & ref. \\
\hline 2012 & $1.53(0.87,2.69)$ \\
\hline \multicolumn{2}{|l|}{ Age at diagnosis } \\
\hline$<50$ years & ref. \\
\hline 50-59 years & $1.49(0.58,3.82)$ \\
\hline 60-69 years & $1.65(0.70,3.91)$ \\
\hline$\geq 70$ years & $1.15(0.46,2.88)$ \\
\hline \multicolumn{2}{|l|}{ Sex } \\
\hline Male & ref. \\
\hline Female & $0.76(0.45,1.28)$ \\
\hline \multicolumn{2}{|l|}{ Race/ethnicity } \\
\hline Non-Hispanic White & ref. \\
\hline Non-Hispanic Black & $1.89(1.03,3.46)$ \\
\hline Hispanic & $1.97(0.88,4.43)$ \\
\hline Asian/other & $1.84(0.92,3.72)$ \\
\hline \multicolumn{2}{|l|}{ Marital status } \\
\hline Married/living as married & ref. \\
\hline Other & $0.71(0.39,1.27)$ \\
\hline \multicolumn{2}{|l|}{ Insurance status } \\
\hline Private insurance & ref. \\
\hline Medicare only & $0.81(0.33,2.01)$ \\
\hline Any Medicaid & $0.67(0.37,1.23)$ \\
\hline No insurance/unknown & $0.35(0.11,1.12)$ \\
\hline \multicolumn{2}{|l|}{ Median census tract income } \\
\hline USD $\leq 50,000 /$ year & ref. \\
\hline USD > 50,000/year & $1.50(0.89,2.55)$ \\
\hline \multicolumn{2}{|l|}{ Hepatitis } \\
\hline Yes & ref. \\
\hline No indication & $1.01(0.55,1.84)$ \\
\hline \multicolumn{2}{|l|}{ Cirrhosis } \\
\hline Yes & ref. \\
\hline No indication & $1.43(0.85,2.41)$ \\
\hline \multicolumn{2}{|l|}{ Child-Pugh score } \\
\hline Class A & ref. \\
\hline Class B & $0.46(0.27,0.79)$ \\
\hline
\end{tabular}

tices was associated with a lower risk of death. Given the limited treatment options for patients with advanced HCC $[6,25,26]$, this study serves as a starting point to understand variation in the treatment with sorafenib.

Prior to the FDA approval of sorafenib, there was no systemic therapy demonstrated to effectively treat individuals with advanced HCC $[6,26]$. Since 2007 , sorafenib has become the recommended first-line systemic treatment in individuals with HCC who are not candidates for curative treatments, including surgical treatment or locoregional therapy [12, 27-31]. These findings were based on the results of 2 phase III, double-blind, placebo-controlled trials, the first of which (the SHARP trial) examined median survival and radiologic progression in a group of 602 individuals with advanced HCC who had not received previous systemic treatment [8]. The SHARP trial found a median survival improvement for those treated with sorafenib versus placebo of 2.8 months (median survival 10.7 months in the sorafenib group vs. 7.9 
Parsons et al.: Adoption of Sorafenib for the Treatment of Advanced-Stage

Hepatocellular Carcinoma in Oncology Practices in the United States

months in the placebo group) [8]. Similar to the demographic composition of our current population-based study, the SHARP trial included a predominantly male $(87 \%)$ population with a median age between 64 and 66 years of age with advanced HCC (82\% BCLC Stage C). However, the trial population included individuals with lower levels of underlying disease, including hepatitis B or C $(<30 \%)$, who were predominantly characterized as Child-Pugh Class A (95-98\%). The second phase III trial of sorafenib versus placebo was conducted in individuals diagnosed within the Asian-Pacific region and found a comparable survival benefit of 2.3 months (median survival 6.5 months in the sorafenib group vs. 4.2 months in the placebo group) [9]. However, compared to our population-based study of individuals diagnosed with advanced HCC in the US, the Asia-Pacific study included individuals younger at diagnosis (median age 51 years) with significantly higher rates of hepatitis viral infection (70-77\% with hepatitis B virus infection), consistent with the earlier age at onset and concentration of more than $50 \%$ of HCC cases in China alone [32]. The population demographics of these randomizedcontrolled studies (i.e., predominantly Child-Pugh Class A) lend support to the trends in our current study, where we identified that individuals with Child-Pugh score Class B were half as likely to receive sorafenib as those with Class A (Table 2). However, we found no significant difference in cirrhosis or hepatitis status, suggesting that adoption of new therapies for advanced HCC has extended beyond the well-selected clinical populations identified in prior clinical trials. These findings will have important implications for future adoption patterns given recent advances in systemic therapy for advanced HCC, where Bruix et al. [33] recently demonstrated in a phase III clinical trial (RESORCE study) that regorafenib provided significant survival benefits for advanced HCC patients who progressed on sorafenib treatment.

What is less clear is the racial/ethnic variation in receipt of sorafenib we identified in our study, with non-Hispanic Blacks almost twice as likely to receive this therapy as non-Hispanic Whites. In our sample, a higher proportion of non-Hispanic White individuals had some more favorable disease prognoses, including a lack of hepatitis or cirrhosis, compared to other racial/ethnic groups (data not shown), which may have allowed for more aggressive therapies, such as transplant. Prior research on the role of race/ethnicity in cancer treatment has demonstrated a complex relationship between access to and use of novel therapies, race, and survival [34]. Specifically, significant racial variation has been demonstrated in both the diagnosis and treatment of HCC, with Black patients being more likely to be diagnosed at a more advanced stage, having lower utilization of surgical management and similar or worse survival compared to White patients [34,35]. Given that these previous studies have demonstrated that diagnosis with more advanced stage and higher rates of comorbid conditions may preclude surgical management, a higher utilization of sorafenib in the non-Hispanic Black population may make sense clinically in a population with few other treatment options. Future research should continue to examine the complex interaction between race/ethnicity and treatment utilization in the US population.

While few demographic or clinical factors predicted sorafenib use, we found significantly improved survival in those treated with sorafenib compared to systemic chemotherapy, radiofrequency ablation, or no treatment after adjusting for clinical and demographic factors. Unadjusted differences in median survival times for individuals treated with sorafenib versus any other treatment showed survival differences ( 4 months' improved survival) consistent with prior phase III clinical trials (Table 3). However, we identified significantly better survival for those treated with transplant relative to sorafenib, likely due to the more favorable prognostic indicators for these individuals, including lower rates of portal invasion and distant metastasis compared to those treated with other therapies. We additionally found worse survival in those older at diagnosis as well as in those with a higher Child-Pugh score, which may indicate worse performance status or potentially lower tolerance for the demonstrated adverse drug reactions, such as hand-foot skin reactions, hypertension, and diarrhea 
Parsons et al.: Adoption of Sorafenib for the Treatment of Advanced-Stage Hepatocellular Carcinoma in Oncology Practices in the United States

Table 3. Association between patient demographic and clinical characteristics and 12-month survival, Cox proportional hazards regression (2007 and 2012 National Cancer Institute Patterns of Care data)

\begin{tabular}{|c|c|c|}
\hline & $\begin{array}{l}\text { Hazard ratio ( } 95 \% \\
\text { confidence interval) }\end{array}$ & $\begin{array}{l}\text { Median survival, } \\
\text { months }\end{array}$ \\
\hline \multicolumn{3}{|l|}{ Year of diagnosis } \\
\hline 2007 & ref. & 4 \\
\hline 2012 & $0.99(0.74,1.33)$ & 4 \\
\hline \multicolumn{3}{|l|}{ Age at diagnosis } \\
\hline$<50$ years & ref. & 8 \\
\hline $50-59$ years & $2.62(1.13,6.10)$ & 4 \\
\hline 60-69 years & $3.12(1.40,6.93)$ & 5 \\
\hline$\geq 70$ years & $2.86(1.29,6.34)$ & 3 \\
\hline \multicolumn{3}{|l|}{ Sex } \\
\hline Male & ref. & 4 \\
\hline Female & $1.05(0.77,1.43)$ & 4 \\
\hline \multicolumn{3}{|l|}{ Race/ethnicity } \\
\hline Non-Hispanic White & ref. & 4 \\
\hline Non-Hispanic Black & $0.97(0.62,1.50)$ & 4 \\
\hline Hispanic & $0.93(0.65,1.33)$ & 5 \\
\hline Asian/other & $1.42(0.99,2.04)$ & 3 \\
\hline \multicolumn{3}{|l|}{ Marital status } \\
\hline Married/living as married & ref. & 4 \\
\hline Other & $0.90(0.61,1.32)$ & 4 \\
\hline \multicolumn{3}{|l|}{ Insurance status } \\
\hline Private insurance & ref. & 5 \\
\hline Medicare only & $1.18(0.81,1.72)$ & 3 \\
\hline Any Medicaid & $0.97(0.60,1.58)$ & 4 \\
\hline No insurance/unknown & $0.89(0.34,2.34)$ & 3 \\
\hline Median census tract income & & 4 \\
\hline USD $\leq 50,000 /$ year & ref. & \\
\hline USD > 50,000/year & $1.03(0.74,1.43)$ & 5 \\
\hline \multicolumn{3}{|l|}{ Treatment received } \\
\hline Any sorafenib & ref. & 7 \\
\hline Any liver transplant & $0.10(0.01,0.77)$ & not reached \\
\hline Surgery only & $0.70(0.30,1.65)$ & 15 \\
\hline Embolization only & $0.79(0.49,1.29)$ & 7 \\
\hline Systemic chemotherapy only & $2.27(1.41,3.66)$ & 3 \\
\hline Radiofrequency ablation only & $4.53(2.18,9.40)$ & 2.5 \\
\hline Multiple therapies & $0.50(0.30,0.83)$ & 10 \\
\hline No treatment & $3.79(2.89,4.96)$ & 2 \\
\hline Unknown treatment & $1.45(0.38,5.60)$ & 1.5 \\
\hline \multicolumn{3}{|l|}{ Hepatitis } \\
\hline Yes & ref. & 4 \\
\hline No indication & $1.44(0.98,2.13)$ & 4 \\
\hline \multicolumn{3}{|l|}{ Cirrhosis } \\
\hline Yes & ref. & 5 \\
\hline No indication & $0.90(0.68,1.18)$ & 4 \\
\hline \multicolumn{3}{|l|}{ Child-Pugh score } \\
\hline Class A & ref. & 6 \\
\hline Class B & $1.43(1.07,1.90)$ & 3 \\
\hline \multicolumn{3}{|l|}{ Sorafenib } \\
\hline Yes & n.a. & 7 \\
\hline No & & 3 \\
\hline
\end{tabular}

Italics indicate $p<0.05$. n.a., not applicable (already adjusted for within the treatment variable). 
$[8,17-20]$. While we were not able to specifically examine the impact of performance status or adverse reactions in our study, future evaluations should focus on the tolerability of sorafenib outside of well-designed clinical trials or academic health settings in order to understand the potential treatment burden in the general population of individuals with advanced HCC [13]. Additionally, considering the estimated USD 10,000-15,000/month in drug-related treatment costs for sorafenib, continuing discussions surrounding the effectiveness of sorafenib relative to costs will be needed to balance this novel treatment opportunity with the potential for side effects and high treatment costs in oncology practices.

While we demonstrated population-based trends in a novel therapy for advanced HCC, we recognize certain limitations. First, we could not determine patients' performance status in the current study. Additionally, we did not have information on the presence of non-alcoholic fatty liver disease or alcohol use. However, our study allowed us to evaluate a broad array of clinical and treatment characteristics, including comorbidities, and the presence of cirrhosis and hepatitis. Comorbidities were collected, and the presence of cirrhosis and hepatitis was used in our analysis. Finally, we did not have information on the clinical management of patients in our study with hepatitis or cirrhosis, which may influence treatment decisionmaking. While these patients may have undergone active surveillance or treatment based on current clinical guidelines, a recent study has identified poor compliance with these recommendations in a large academic health center [36]. Overall, this large, population-based sample of individuals with advanced HCC can serve as a starting point for understanding the adoption of new therapies in this complex population.

In conclusion, despite these limitations, our large population-based study of individuals diagnosed with advance HCC in the US demonstrates that sorafenib treatment has not been widely adopted into oncology practice since FDA approval in 2007. Further, few factors apart from the Child-Pugh score and race/ethnicity predict sorafenib use in clinical practice, although sorafenib treatment is associated with a lower risk of death. Our findings go beyond the initial efficacy trials which included only well-selected clinical populations [8,9] and provide a first look at limited sorafenib adoption patterns and associated survival in the general oncology population throughout the US. Our findings can be used to further the discussion surrounding appropriate adoption of sorafenib in light of ongoing discussions of promoting value in cancer care.

\section{Acknowledgement}

The authors would like to acknowledge the work of the SEER Cancer Registries. This research would not have been possible without their efforts.

\section{Disclosure Statement}

The authors have no conflicts of interest to declare.

\section{Funding Sources}

Dr. Parsons receives support from an NCI Cancer Prevention and Control Career Development Award (K07CA175063). Supported by NCI Contracts HHSN261201000024C, HHSN261201000025C, HSC261201000032C, HHSN261201000027C, HHSN261201000026C, HHSN261201000140C, HHSN261201000037C, HHSN261201000033C, HHSN261201000034C, HHSN261201000035C, HHSN261201000029C, HHSN261201000031C, HHSN261201000028C, and HHSN261201000030C.

This article is a US Government work and, as such, is in the public domain in the United States of America. 
Parsons et al.: Adoption of Sorafenib for the Treatment of Advanced-Stage

Hepatocellular Carcinoma in Oncology Practices in the United States

\section{References}

1 Venook AP, Papandreou C, Furuse J, de Guevara LL: The incidence and epidemiology of hepatocellular carcinoma: a global and regional perspective. Oncologist 2010;15(suppl 4):5-13.

2 El-Serag HB: Hepatocellular carcinoma. N Engl J Med 2011;365:1118-1127.

3 American Cancer Society: Cancer Facts and Figures 2015. 2015. http://www.cancer.org/acs/groups/ content/@editorial/documents/document/acspc-044552.pdf (accessed June 1, 2015).

4 El-Serag HB, Marrero JA, Rudolph L, Reddy KR: Diagnosis and treatment of hepatocellular carcinoma. Gastroenterology 2008;134:1752-1763.

5 Chiu J, Tang YF, Yao TJ, et al: The use of single-agent sorafenib in the treatment of advanced hepatocellular carcinoma patients with underlying Child-Pugh B liver cirrhosis: a retrospective analysis of efficacy, safety, and survival benefits. Cancer 2012;118:5293-5301.

6 Salhab M, Canelo R: An overview of evidence-based management of hepatocellular carcinoma: a meta-analysis. J Cancer Res Ther 2011;7:463-475.

7 National Cancer Institute: FDA Approval for Sorafenib Tosylate. 2015. http://www.cancer.gov/about-cancer/ treatment/drugs/fda-sorafenib-tosylate (accessed June 8, 2015).

8 Llovet JM, Ricci S, Mazzaferro V, et al: Sorafenib in advanced hepatocellular carcinoma. N Engl J Med 2008;359: 378-390.

9 Cheng AL, Kang YK, Chen Z, et al: Efficacy and safety of sorafenib in patients in the Asia-Pacific region with advanced hepatocellular carcinoma: a phase III randomised, double-blind, placebo-controlled trial. Lancet Oncol 2009;10:25-34.

10 National Institute for Health and Care Excellence: Sorafenib for the Treatment of Advanced Hepatocellular Carcinoma. 2015. https://www.nice.org.uk/guidance/ta189 (accessed June 8, 2015).

11 National Comprehensive Cancer Network: NCCN Clinical Practice Guidelines in Oncology: Hepatobiliary Cancers. 2015. http://www.nccn.org/professionals/physician_gls/pdf/hepatobiliary.pdf (accessed June 9, 2015).

12 Bruix J, Sherman M: Management of hepatocellular carcinoma: an update. Hepatology 2011;53:1020-1022.

13 Federico A, Orditura M, Cotticelli G, et al: Safety and efficacy of sorafenib in patients with advanced hepatocellular carcinoma and Child-Pugh A or B cirrhosis. Oncol Lett 2015;9:1628-1632.

14 Ogasawara S, Chiba T, Ooka Y, et al: Sorafenib treatment in Child-Pugh A and B patients with advanced hepatocellular carcinoma: safety, efficacy and prognostic factors. Invest New Drugs 2015;33:729-739.

15 Bach PB: Limits on Medicare's ability to control rising spending on cancer drugs. N Engl J Med 2009;360: 626-633.

16 Wilson A, Cohen J: Patient access to new cancer drugs in the United States and Australia. Value Health 2011; 14:944-952.

17 Robert C, Soria JC, Spatz A, et al: Cutaneous side-effects of kinase inhibitors and blocking antibodies. Lancet Oncol 2005;6:491-500.

18 Ratain MJ, Eisen T, Stadler WM, et al: Phase II placebo-controlled randomized discontinuation trial of sorafenib in patients with metastatic renal cell carcinoma. J Clin Oncol 2006;24:2505-2512.

19 Knowledge Ecology International: Sorafenib (Nexavar). 2016. http://keionline.org/prices/nexavar (accessed January 29, 2015).

20 Q1 Group LLC: 2016 Drug Finder: Medicare Part D or Medicare Advantage Plans. 2016. http://www. q1medicare.com/PartD-SearchPDPMedicarePartDDrugFinder.php?stateReg=32CA\&plans=Humana\&plans2 $=$ AdvantraRx\&sort=\&ded=\&gap=\&prem=\&ndc=50419048858\&drug=NEXAVAR (accessed January 29, 2016).

21 National Cancer Institute: Patterns of Care/Quality of Care Studies. 2016. http://healthcaredelivery.cancer. gov/poc/ (accessed January 29, 2016).

22 National Cancer Institute: Overview of the SEER Program. 2016. http://seer.cancer.gov/about/overview.html (accessed January 28, 2016).

23 Durand F, Valla D: Assessment of the prognosis of cirrhosis: Child-Pugh versus MELD. J Hepatol 2005; 42(suppl):S100-S107.

24 Llovet JM, Bru C, Bruix J: Prognosis of hepatocellular carcinoma: the BCLC staging classification. Semin Liver Dis 1999;19:329-338.

25 Alves RC, Alves D, Guz B, et al: Advanced hepatocellular carcinoma. Review of targeted molecular drugs. Ann Hepatol 2011;10:21-27.

26 Keating GM, Santoro A: Sorafenib: a review of its use in advanced hepatocellular carcinoma. Drugs 2009;69: 223-240.

27 Flores A, Marrero JA: Emerging trends in hepatocellular carcinoma: focus on diagnosis and therapeutics. Clin Med Insights Oncol 2014;8:71-76.

28 Morimoto M, Numata K, Kondo M, et al: Higher discontinuation and lower survival rates are likely in elderly Japanese patients with advanced hepatocellular carcinoma receiving sorafenib. Hepatol Res 2011;41:296302.

29 Bruix J, Takayama T, Mazzaferro V, et al: Adjuvant sorafenib for hepatocellular carcinoma after resection or ablation (STORM): a phase 3, randomised, double-blind, placebo-controlled trial. Lancet Oncol 2015;16: 1344-1354. 


\section{Liver Cancer}

\begin{tabular}{l|l}
\hline \multicolumn{2}{l|}{ Liver Cancer 2017;6:216-226 } \\
\hline DOI: 10.1159/000473862 & $\begin{array}{l}\text { C 2017 S. Karger AG, Basel } \\
\text { www.karger.com/lic }\end{array}$ \\
\hline
\end{tabular}

Parsons et al:: Adoption of Sorafenib for the Treatment of Advanced-Stage Hepatocellular Carcinoma in Oncology Practices in the United States

30 European Association for the Study of the Liver; European Organisation for Research and Treatment of Cancer: EASL-EORTC clinical practice guidelines: management of hepatocellular carcinoma. J Hepatol 2012; 56:908-943.

31 Omata M, Lesmana LA, Tateishi R, et al: Asian Pacific Association for the Study of the Liver consensus recommendations on hepatocellular carcinoma. Hepatol Int 2010;4:439-474.

32 Jemal A, Bray F, Center MM, Ferlay J, Ward E, Forman D: Global cancer statistics. CA Cancer J Clin 2011;61: 69-90.

33 Bruix J, Qin S, Merle P, et al: Regorafenib for patients with hepatocellular carcinoma who progressed on sorafenib treatment (RESORCE): a randomised, double-blind, placebo-controlled, phase 3 trial. Lancet 2017; 389:56-66.

34 Hoehn RS, Hanseman DJ, Wima K, et al: Does race affect management and survival in hepatocellular carcinoma in the United States? Surgery 2015;158:1244-1251.

35 Jan T, Medvedev S, Cannon RM, et al: Racial disparity and their impact on hepatocellular cancer outcomes in inner-city New Orleans. Surgery 2012;152:661-666; discussion 666-667.

$36 \mathrm{Wu}$ Y, Johnson KB, Roccaro G, et al: Poor adherence to AASLD guidelines for chronic hepatitis B management and treatment in a large academic medical center. Am J Gastroenterol 2014;109:867-875. 